\title{
Duplex Surveillance after Primary Balloon Angioplasty or Directional Atherectomy for Treating Infrainguinal Arterial Disease
}

\author{
Kyung Pyo Hong, Sungsin Cho, Jin Hyun Joh \\ Department of Surgery, Kyung Hee University Hospital at Gangdong, Kyung Hee University School of Medicine, Seoul, Korea
}

Received October 5, 2019

Revised November 18, 2019

Accepted November 26, 2019

\section{Correspondence to:}

Jin Hyun Joh

Department of Surgery, Kyung Hee

University Hospital at Gangdong,

Kyung Hee University School of

Medicine, 892 Dongnam-ro,

Gangdong-gu, Seoul 05278, Korea

Tel: + 82-2-440-6261

Fax: +82-2-440-6296

E-mail: jhjoh@khu.ac.kr
Purpose: Percutaneous transluminal angioplasty (PTA) shows an attractive outcome for treating infrainguinal arterial occlusive disease (IAOD). However, this procedure requires repeated revascularization and additional stenting. Directional atherectomy (DA) has been reported to be a modality that requires less frequent use of stenting. The aim of this study was to compare the duplex-based outcomes between PTA and DA for treating IAOD.

Methods: A retrospective review of consecutive patients with IAOD treated with PTA and DA was completed. The demographics and procedural data were obtained. A provisional stent was placed in those cases with flow-limiting dissection and residual stenosis after PTA or DA. Technical success was defined as a residual stenosis $<30 \%$. The primary patency rate was evaluated by performing duplex scanning. The clinical outcomes and frequency of stent placement for each procedure were evaluated. For statistical analysis, the data was analyzed using SPSS 22.0 software (IBM Corp, Chicago, Ill). All P values were considered significant if less than 0.05 .

Results: Seventy-one patients were included in the study. Their mean age was $71.5 \pm 10.2$ years. The male-to-female ratio was 49:22. Thirty-three patients underwent PTA. DA was performed in 38 patients (42 limbs). Technical success was achieved in all the patients. The duplex-based clinical outcome and limb salvage rate were similar in the two groups. Bailout stent placement was performed in 8 of 33 patients $(24.2 \%)$ in the PTA group and in no patient in the DA group.

Conclusion: The clinical outcomes were similar for both the DA and primary PTA groups. Atherectomy reduced the need for bailout stent placement as compared with bailout stent placement in primary PTA.

Keywords: Transluminal angioplasty, Percutaneous atherectomy, Atherosclerosis, Stent, Duplex scan

\section{INTRODUCTION}

Infrainguinal arterial occlusive disease (IAOD) is a common vascular problem. It carries the decreased quality of life and possible major limb loss.(1)
Traditionally, the open bypass surgery has been a standard for its treatment. However, a variety of endovascular procedure is an increasingly performed for its treatment. $(2,3)$ At many centers, the preferred treatment of infrainguinal occlusive disease

Journal of Surgical Ultrasound is an Open Access Journal. All articles are distributed under the terms of the Creative Commons Attribution Non-Commercial License (http://creativecommons org/licenses/by-nc/4.0/) which permits unrestricted non-commercial use, distribution, and reproduction in any medium, provided the original work is properly cited. 
has undergone a paradigm shift from open bypass surgery to endovascular intervention as the firstline therapeutic modality.

The percutaneous transluminal angioplasty (PTA) can be a core of endovascular therapy. PTA can be a definite treatment modality or be performed as an initial procedure for other endovascular procedure. Although PTA can dilate the steno-occlusive arterial lesion, the plaque itself may be split or fractured, but otherwise does not change significantly in volume or shape.(4) Therefore, PTA showed provisional stent placement rates ranges up to 50\%. (5) Although the newer nitinol-self expanding stents have increased conformability and low fracture rates, in-stent restenosis (ISR) remains a common clinical problem, with an estimated rate of $19 \%-37 \%$. $(6,7)$ Directional atherectomy has been used to treat the infrainguinal PAOD as the strategies 'NOTHING LEFT BEHIND'. (8)

There has not been reported the detailed or comprehensive strategies that specified the optimal approaches with regard to testing methods after the endovascular revascularization for the IAOD. The Clinical Practice Council of the Society for Vascular Surgery suggested that ankle-brachial index (ABI) and duplex ultrasound (DUS) within the first month to provide a post-treatment baseline and to evaluate for residual stenosis after intervention of IAOD and continued surveillance at 3 months and then every 6 months. (9) The purpose of this study was to compare the clinical outcomes after PTA and DA for IAOD.

\section{METHODS}

Consecutive patients from the Kyung Hee University Hospital at Gangdong (Seoul, Korea) were selected during the study period. Written informed consent for the procedure was obtained from all the patients or their families. Each patient had disabling claudication or critical limb ischemia (Rutherford category 2 to 6 ) and underwent revascularization by
PTA or DA. The patients were retrospectively analyzed using a prospectively collected database.

Demographic and clinical characteristics of the patients were recorded, including comorbidities (hypertension, diabetes, smoking, coronary artery disease, cerebrovascular disease, chronic obstructive pulmonary disease, hyperlipidemia and chronic renal failure), clinical presentation, and the clinical outcomes on last follow-up. Preoperatively, all patients underwent computed tomography-angiography (CTA) for the evaluation of lower extremity arterial occlusive lesions. Treatment modality was determined by the vascular surgeon based on the CTA images.

All subjects underwent revascularization of target lesions of the femoropopliteal and/or tibial-peroneal arteries with PTA or DA using a DA device. If the DA device could not initially pass through the lesion, pre-dilation was performed to facilitate DA device passage. Adjunctive procedures including PTA was performed per physician discretion; however, if residual stenosis was $<30 \%$, additional post-dilation was not performed.

Technical success for an endovascular procedure was defined as a patent vessel with $<30 \%$ residual stenosis, following postdilatation with restoration of rapid antegrade perfusion. Postoperative surveillance was performed in outpatient clinics within one month and at 3, 6 months and every 6 months thereafter. Clinical assessments of the femoral and distal pulses, ABI measurements and DUS were performed. Primary patency was defined as duplex ultrasonography measurement of the peak systolic velocity ratio $($ PSVR) $<2.5$ at the target lesion. The occlusion of the target lesion was defined as the no color flow image and no signal by the spectral Doppler image. The independent t-test for continuous variables, Fisher's exact test for categorical variables, and Log-rank test for patency rate using SPSS 22.0 software (IBM Corp, Chicago, Ill) were used for statistical analysis. All $\mathrm{P}$ values were 
2-tailed, and $\mathrm{P}<0.05$ was considered to be statistically significant.

\section{RESULTS}

A total of 71 patients were included in the study. The flow chart for the enrollment of patients was shown in Fig. 1. We analyzed the patients who underwent the endovascular revascularization of IAOD from the prospectively collected database of peripheral arterial occlusive disease of 413 patients. The patients with upper extremity disease or acute arterial disease were excluded. And the aortic and iliac arterial procedures were excluded. Then, the remaining 71 patients who underwent endovascular procedures for the IAOD were included to this study.

Demographic and preoperative characteristics of the patients are shown in Table 1 . The mean age was $70.3 \pm 7.8$ years in PTA and/or stenting group and $72.5 \pm 11.9$ years in DA group, respectively. The male-to-female ratio was 49:22. The most common indication for revascularization was Rutherford category V (minor tissue loss) and hypertension was the most common comorbidity in both groups. The length of follow-up period was significantly longer in PTA group than DA group (20.3 months versus 5.3 months, $\mathrm{P}=0.001)$. The most common anatomic characteristics according to the TASC classification showed the TASC B lesion. Technical success was achieved in all patients. One of interesting point was that there was no case of bail-out stenting in DA group.

The duplex surveillance was performed within one months and at 3, 6 months and every 6 months thereafter. Primary patency rate defined as a peak systolic velocity ratio $<2.4$ was shown in Fig. 2. One-year primary patency rate was $78.1 \%$ in PTA group and $89.8 \%$ in DA group $(\mathrm{P}=0.125)$. Two-year primary patency rate was $71.6 \%$ in PTA group. The primary patency rate according to the lesion length was shown in Fig. 3.

\section{DISCUSSION}

We report on the short-term results of a retrospective cohort patients with infrainguinal lesions treated with PTA and DA. Technical success rate was $100 \%$. Although the follow-up period was relatively

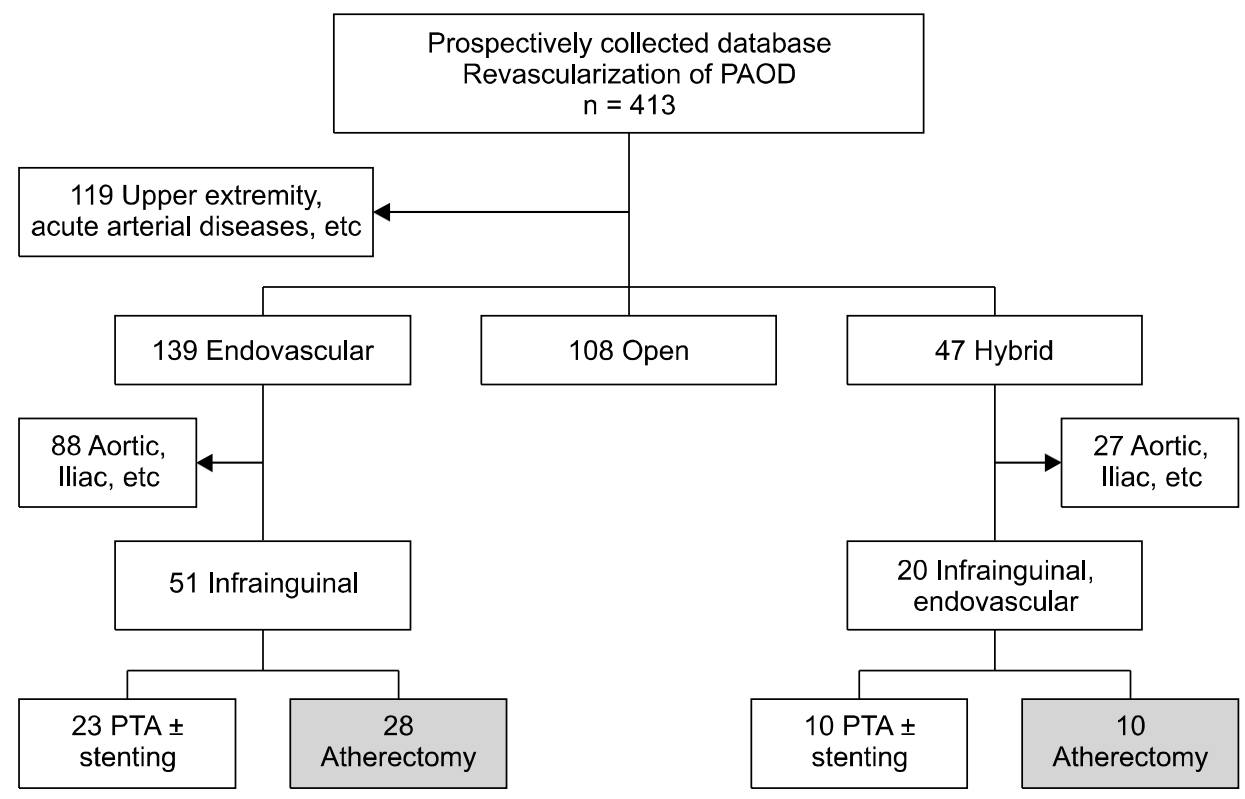

Fig. 1. Flow chart of the procedures performed during the study period. 
Table 1. Patient Characteristics

\begin{tabular}{lccc}
\hline \multicolumn{1}{c}{ Characteristics } & PTA \pm Stenting & Atherectomy & P value* \\
\hline Number & 33 & 38 & - \\
Age, years & $70.3 \pm 7.8$ & $72.5 \pm 11.9$ & 0.374 \\
Sex (male:female) & $21: 12$ & $28: 10$ & 0.443 \\
Follow-up, months & $20.3 \pm 16.6$ & $5.3 \pm 4.3$ & 0.001 \\
Rutherford category (2/3/4/5/6) & $1 / 12 / 0 / 14 / 6$ & $0 / 11 / 1 / 20 / 6$ & 0.733 \\
TASC classification (A:B:C:D) & $12: 14: 5: 2$ & $14: 16: 8: 0$ & 0.560 \\
Bail-out stenting (yes:no) & $11: 22$ & $0: 38$ & 0.001 \\
Risk factors & & & 0.190 \\
Hypertension & $21(63.6 \%)$ & $30(78.9 \%)$ & 0.477 \\
Diabetes & $14(42.4 \%)$ & $20(52.6 \%)$ & 0.002 \\
Smoking & $20(60.6 \%)$ & $9(23.7 \%)$ & 1.00 \\
Coronary artery disease & $7(21.2 \%)$ & $8(21.1 \%)$ & 0.383 \\
Cerebrovascular disease & $5(15.2 \%)$ & $10(26.3 \%)$ & 0.212 \\
COPD & $2(6.1 \%)$ & 0 & 0.742 \\
Hyperlipidemia & $4(12.1 \%)$ & $6(15.8 \%)$ & 1.00 \\
Chronic renal failure & $8(24.2 \%)$ & $10(26.3 \%)$ & 0.697 \\
Mortality (all causes) & $4(12.1 \%)$ & $3(7.9 \%)$ & \\
\hline
\end{tabular}

PTA $=$ percutaneous transluminal angioplasty; TASC $=$ transathlantic inter-societal consensus; COPD $=$ chronic obstructive pulmonary disease. *Statistical analysis was done using independent t-test for comparison of continuous variables and Fisher's exact test for comparison of nominal variables.

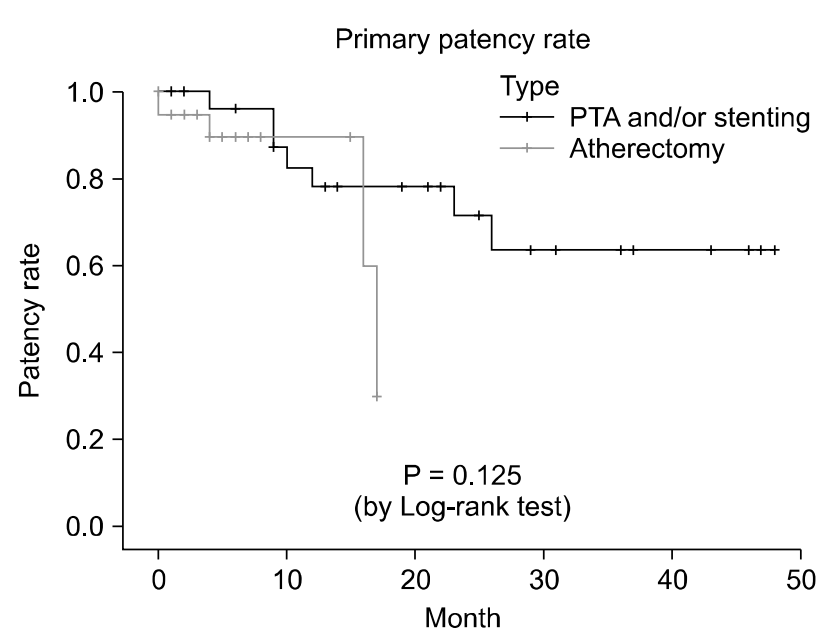

\begin{tabular}{llccccc}
\hline Months (patency rate \%) & 0 & 3 & 6 & 12 & 24 \\
\hline Number & PTA \pm stenting & 33 & $33(100)$ & $22(96.0)$ & $17(78.1)$ & $10(71.6)$ \\
at risk & Atherectomy & 38 & $19(94.7)$ & $8(89.8)$ & $3(89.8)$ & - \\
\hline
\end{tabular}

Fig. 2. Primary patency rate measured by duplex scanning of two groups. PTA = percutaneous transluminal angioplasty.

short, the primary patency rate was $78.1 \%$ in in PTA group and $89.8 \%$ in DA group. In most of cases in DA group, additional ballooning was performed to achieve the better angiographic appearance. Zeller et al. (10) also reported that atherectomy alone in the

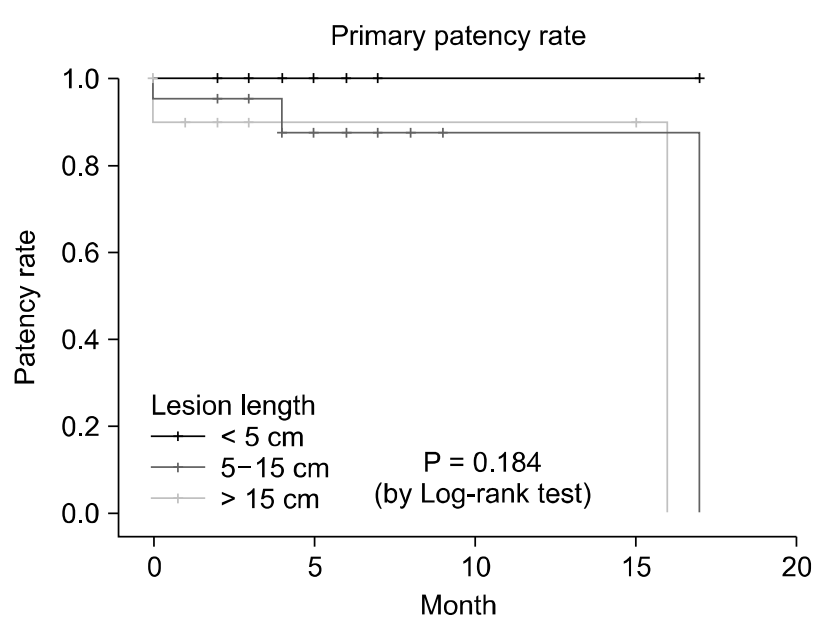

\begin{tabular}{lccccc}
\hline Months (patency \%) & 0 & 3 & 6 & 12 \\
\hline Number & $<5 \mathrm{~cm}$ & $10(100)$ & $8(100)$ & $4(100)$ & $1(100)$ \\
at risk & $5-15 \mathrm{~cm}$ & $22(100)$ & $12(90.5)$ & $4(87.5)$ & - \\
& $>15 \mathrm{~cm}$ & $10(100)$ & $2(90.0)$ & $1(90.0)$ & $1(90.0)$ \\
\hline
\end{tabular}

Fig. 3. Primary patency rate measured by duplex scanning depending on the lesion length.

majority (96\%) of cases was technically successful, but additional balloon dilation in; 60\% of cases served to visually improve the angiographic appearance of the recanalized artery. Only about 24\% required dilation to achieve primary treatment success. 
From a technical view, the DA catheter could easily be controlled in both antegrade and crossover use, despite wire guidance of the monorail system over only a short distance. The few occasions in which the blade became temporarily locked in the forward position occurred primarily when the vessel had a bend or turn. For the case of up-and-over technique to cross the aortic bifurcation, this sort of situation occurred. This problem was managed by the change of sheath with a stiffer sheath such as a 7F Balkin sheath (Cook Inc., Bloomington, IN, USA). The blade could always be easily freed. The atherectomy catheter we tested in this study was limited to lesions with slight degree of calcification because the rotating stainless steel blade did not cut through pure calcium.(10)

One of troublesome complication was the distal embolization at the beginning of our experience. The DEFINITIVE $\mathrm{Ca}^{++}$study showed the $2.3 \%$ of this complication. They recommended the use of distal embolic protection device.(11) The value of using of embolic protection with atherectomy is controversial and varies by lesion characteristics and disease severity. The presence of embolization has been shown across all endovascular modalities (PTA, stent, laser and atherectomy) using embolic filters $(12,13)$ or by continuous monitoring of the downstream artery with duplex ultrasound. (14) Furthermore, calcification was shown to increase the risk of clinically significant embolism. (15)

DUS is commonly used to evaluate the clinical outcomes or primary patency ate after endovascular procedure of IAOD. Investigators at the University of Pittsburgh Medical Center conducted a retrospective review of 330 limbs that underwent stenting of the superficial femoral artery. These patients were seen in follow-up at 1 month, 3 months, 6 months, and every 6 months thereafter. Follow-up included clinical evaluation, ABI measurement, and DUS. Criteria for high-grade restenosis were PSV $>300 \mathrm{~cm} / \mathrm{s}$ and $\mathrm{Vr}>$ 3.5.(16) Shrikhande et al.(17) reported a similar surveillance protocol with follow-up at 1 month, 3 months, and 6 months and yearly thereafter. They concluded that a PSV $>223 \mathrm{~cm} / \mathrm{s}$ had both high specificity and sensitivity in the femoropopliteal segment to detect a $>70 \%$ stenosis. Using a $\mathrm{Vr}>2.5$ also identified a high percentage of patients with a $>70 \%$ angiographic stenosis. Bui et al.(18) investigated the natural history of restenosis in the femoropopliteal segment after EVT. Ninety-four limbs were entered into a DUS surveillance protocol after EVT, and the mean follow-up was 22 months. Moderate stenosis was defined by a PSV of 200 to 300 $\mathrm{cm} / \mathrm{s}$ or a Vr of 2 to 3 , and severe stenosis was defined by a PSV $>300 \mathrm{~cm} / \mathrm{s}$ or a $\mathrm{Vr}>3$.

The endovascular procedure in the tibial arteries is associated with low patency rates and high restenosis rates. $(19,20)$ Saqib et al. (21) reported on the outcomes and predictors of restenosis for EVT of the tibial arteries. A PSV $>300 \mathrm{~cm} / \mathrm{s}$ and $\mathrm{Vr}$ of $>3.5$ were used as indicators of severe restenosis. There have been conflicting reports about the utility of DUS after EVT in the tibial arteries, with some authors finding poor correlation to angiographic findings, (17) whereas others have found it to be reliable for surveillance after interventions. (22)

Our analysis has several limitations. This is the retrospective data collection based on administrative database. Another limitation is short-term follow-up period. Furthermore, this is the relatively small sample size. Further studies have to examine the best use of the Silverhawk atherectomy device regarding long term patency. Potential biases could have occurred in that clinicians at the practice sites were aware of the goals of the study and therefore may have preferentially overenrolled patients with known PAOD. Also patients with leg symptoms may have been more willing to participate. 


\section{CONCLUSION}

The directional atherectomy is an effective treatment modality at 12 months for the patients with IAOD. The procedural success can be achieved without a provisional stent placement after DA.

\section{CONFLICTS OF INTEREST}

None.

\section{REFERENCES}

1. Hirsch AT, Criqui MH, Treat-Jacobson D, Regensteiner JG, Creager MA, Olin JW, et al. Peripheral arterial disease detection, awareness, and treatment in primary care. JAMA 2001;286:1317-24.

2. Goodney PP, Beck AW, Nagle J, Welch HG, Zwolak RM. National trends in lower extremity bypass surgery, endovascular interventions, and major amputations. J Vasc Surg 2009;50:54-60.

3. Park YY, Joh JH, Han SA, Kim SH, Cho S, Park HC, et al. National trends for the treatment of peripheral arterial disease in Korea between 2004 and 2013. Ann Surg Treat Res 2015;89:319-24.

4. Gardiner GA Jr, Bonn J, Sullivan KL. Quantification of elastic recoil after balloon angioplasty in the iliac arteries. J Vasc Interv Radiol 2001;12:1389-93.

5. Shammas NW, Coiner D, Shammas GA, Dippel EJ, Christensen L, Jerin M. Percutaneous lower-extremity arterial interventions with primary balloon angioplasty versus Silverhawk atherectomy and adjunctive balloon angioplasty: randomized trial. J Vasc Interv Radiol 2011;22:1223-8.

6. Schillinger M, Sabeti S, Loewe C, Dick P, Amighi J, Mlekusch W, et al. Balloon angioplasty versus implantation of nitinol stents in the superficial femoral artery. N Engl J Med 2006;354:1879-88.

7. Laird JR, Katzen BT, Scheinert D, Lammer J, Carpenter J, Buchbinder M, et al.; RESILIENT Investigators. Nitinol stent implantation versus balloon angioplasty for lesions in the superficial femoral artery and proximal popliteal artery: twelve-month results from the RESILIENT randomized trial. Circ Cardiovasc Interv 2010;3:267-76

8. Garcia LA, Lyden SP. Atherectomy for infrainguinal peripheral artery disease. J Endovasc Ther 2009;16(2 Suppl 2):II105-15.

9. Zierler RE, Jordan WD, Lal BK, Mussa F, Leers S, Fulton J, et al. The Society for Vascular Surgery practice guidelines on follow-up after vascular surgery ar- terial procedures. J Vasc Surg 2018;68:256-84.

10. Zeller T, Rastan A, Schwarzwälder U, Frank U, Bürgelin K, Amantea P, et al. Percutaneous peripheral atherectomy of femoropopliteal stenoses using a new-generation device: six-month results from a single-center experience. J Endovasc Ther 2004;11: $676-85$.

11. Roberts D, Niazi K, Miller W, Krishnan P, Gammon R, Schreiber T, et al. Effective endovascular treatment of calcified femoropopliteal disease with directional atherectomy and distal embolic protection: final results of the DEFINITIVE $\mathrm{Ca}^{++}$trial. Catheter Cardiovasc Interv 2014;84:236-44.

12. Shammas NW, Dippel EJ, Coiner D, Shammas GA, Jerin M, Kumar A. Preventing lower extremity distal embolization using embolic filter protection: results of the PROTECT registry. J Endovasc Ther 2008;15: $270-6$.

13. Shammas NW, Coiner D, Shammas GA, Christensen L, Dippel EJ, Jerin M. Distal embolic event protection using excimer laser ablation in peripheral vascular interventions: results of the DEEP EMBOLI registry. J Endovasc Ther 2009;16:197-202.

14. Lam RC, Shah S, Faries PL, McKinsey JF, Kent KC, Morrissey NJ. Incidence and clinical significance of distal embolization during percutaneous interventions involving the superficial femoral artery. J Vasc Surg 2007;46:1155-9.

15. Davies MG, Bismuth J, Saad WE, Naoum JJ, Mohiuddin IT, Peden EK, et al. Implications of in situ thrombosis and distal embolization during superficial femoral artery endoluminal intervention. Ann Vasc Surg 2010;24:14-22.

16. Baril DT, Rhee RY, Kim J, Makaroun MS, Chaer RA, Marone LK. Duplex criteria for determination of in-stent stenosis after angioplasty and stenting of the superficial femoral artery. J Vasc Surg 2009;49: 133-8; discussion 139.

17. Shrikhande GV, Graham AR, Aparajita R, Gallagher KA, Morrissey NJ, McKinsey JF, et al. Determining criteria for predicting stenosis with ultrasound duplex after endovascular intervention in infrainguinal lesions. Ann Vasc Surg 2011;25:454-60.

18. Bui TD, Mills JL Sr, Ihnat DM, Gruessner AC, Goshima KR, Hughes JD. The natural history of duplex-detected stenosis after femoropopliteal endovascular therapy suggests questionable clinical utility of routine duplex surveillance. J Vasc Surg 2012;55: 346-52.

19. Smolock CJ, Anaya-Ayala JE, Kaufman Y, Bavare CS, Patel MS, El-Sayed HF, et al. Current efficacy of open and endovascular interventions for advanced superficial femoral artery occlusive disease. J Vasc Surg 2013;58:1267-75. e1-2.

20. Graziani L, Piaggesi A. Indications and clinical outcomes for below knee endovascular therapy: review 
article. Catheter Cardiovasc Interv 2010;75:433-43.

21. Saqib NU, Domenick N, Cho JS, Marone L, Leers S, Makaroun MS, et al. Predictors and outcomes of restenosis following tibial artery endovascular interventions for critical limb ischemia. J Vasc Surg
2013;57:692-9.

22. Fernandez N, McEnaney R, Marone LK, Rhee RY, Leers S, Makaroun M, et al. Predictors of failure and success of tibial interventions for critical limb ischemia. J Vasc Surg 2010;52:834-42. 